\title{
Combining Models for Interactive System Modelling
}

Judy Bowen and Steve Reeves

\begin{abstract}
Our approach for modelling interactive systems has been to develop models for the interface and interaction which are light-weight but with an underlying formal semantics. Combined with traditional formal methods to describe functional behaviour, this provides the ability to create a single formal model of interactive systems and consider all parts (functionality, user interface and interaction) with the same rigorous level of formality. The ability to convert the different models we use from one notation to another has given us a set of models which describe an interactive system (or parts of that system) at different levels of abstraction in ways most suitable for the domain but which can be combined into a single model for model-checking, theorem proving etc. There are, however, many benefits to using the individual models for different purposes throughout the development process. In this chapter we provide examples of this using the nuclear power plant control system as an example.
\end{abstract}

\section{Introduction}

Safety-critical interactive systems are software or hardware devices (containing software) operating in an environment where incorrect use or failure may lead to loss, serious harm or death, for example banking systems, ATMs, medical devices, aircraft cockpit software, nuclear power plant control systems, factory production cells etc. Avoiding such errors and harm relies on the systems being developed using robust engineering techniques to ensure that they will behave correctly and also that they can be used successfully.

Judy Bowen

University of Waikato, New Zealand e-mail: jbowen@waikato.ac.nz

Steve Reeves

University of Waikato, New Zealand e-mail: stever@waikato.ac.nz 
Developing suitable interfaces for safety-critical systems requires two things. First, they must be usable in their environments by their users - i.e. they must be developed using a sound user-centred design (UCD) process and following known HCI principles. Secondly, we must be able to verify and validate the user interface and interaction with the same rigour as the underlying functionality. While we can (we hope) assume the former, the latter is harder, and requires us to develop suitable techniques which not only support these requirements but which will also be useful (and used) by the interface developers of such systems.

We have developed modelling techniques for the user interface (UI) and interactivity of a system which take as a starting point typical informal design artefacts which are produced as part of a UCD process, e.g. prototypes (at any level of fidelity), scenarios, storyboards etc. In addition to the interface modelling techniques we also have mechanisms for combining these models with more traditional functional specifications (which deal with the requirements for the system behaviour) in order to be able to reason about the system as a whole.

In the rest of this chapter we provide details of the models and notations we use to describe the different parts of an interactive system. We also discuss how these can be combined into a single model to give a single, formal 'view' of the entire system. This cohesive model allows us to consider important properties of the system (which generally involves proving safety properties and ensuring the system as specified will behave in known, safe ways at all times) which encompasses aspects of the UI and interaction as well as functional behaviour. At the same time, however, the individual component models used to create this single model have their own benefits. They give us the ability to consider different aspects of the system (either specific parts or different groups of behaviours for example) using different levels of abstraction or different modes of description to suit the domain. Essentially they provide us with a set of options from which we can select the most appropriate model for a given use. Because these component models are developed as part of the design process and form part of the overall system model we essentially get this 'for free' (that is, without additional workload).

We use the nuclear power plant control system as an example to show how these models can be used independently, as well as in combination, to consider different properties of interest during a development process.

\section{Related Work}

In early years formal methods were developed as a way of specifying and reasoning about the functionality of systems which did not have the sorts of rich graphical user interfaces provided by today's software. Some formal methods were used to reason about interaction properties, e.g. (Jacob, 1982; Dix and Runciman, 1985), but as user interfaces evolved and became more complex, and the importance of their design became increasingly obvious, the disciplines of HCI and UCD evolved to reflect this. However, these two strands of research - formal system develop- 
ment and UI design research - remained primarily separate and the approaches used within them were also very different. On the one hand were formal languages and notations based on mathematical principles used to formally reason about a system's behaviour via specification, proof, theorem-proving, model-checking etc., while on the other were design processes targetted at usability based on psychological principles and involving shared, informal design artefacts, understanding end-users and their tasks, evaluation of options and usability etc.

This gap between the formal and informal has been discussed many times, notably as far back as 1990 by Thimbleby (Thimbleby, 1990). Numerous approaches have been taken over the years to try and reduce the gap between the two fields, particularly as the need to reason about properties of UIs has become increasingly necessary due to the prevalence of interactive systems in general and the increase in safety-critical interactive systems in particular. We can generalise key works in this area into the following categories:

- development of new formal methods specifically for UIs e.g. (Puerta and Eisenstein, 2002; Courtney, 2003; Limbourg et al, 2004)

- development of hybrid methods from existing formal methods and/or informal design methods. e.g., (Duke and Harrison, 1995; Paternò et al, 1995)

- the use of existing formal methods to describe UIs and UI behaviour (Harrison and Dix, 1990; Thimbleby, 2004)

- replacing existing human-centred techniques with formal model-based methods. e.g. (Hussey et al, 2000; Paternò, 2001; Reichart et al, 2008)

These, and other similar works, constitute a concerted effort and a step forward in bringing formal methods and UI design closer together. However, in many cases the resulting methods, models and techniques continue to either retain the separation of UI and functionality in all stages of the development process, or seek to integrate them by creating new components within the models which combine elements of both in a new way (Duke et al, 1994, 1999).

When we first began to consider the problem and investigate and develop modelling techniques for interactive systems we had a number of criteria, including a desire to model at the most natural level of granularity (describe the existing components as they appear) as well as come up with an approach that could fit with both formal and HCI methodologies. In contrast to other approaches our starting point is that of design artefacts (of both interface and system) which may be developed separately, and perhaps at different times, during the development lifecycle. Unlike more recent work such as (Bolton and Bass, 2010) we do not include models of user behaviour or consider the UI in terms of the tasks performed or user goals. Rather we model at a higher level of abstraction which enables us to consider any available behaviours of the system via the UI rather than constrain this to expected actions of the user based on pre-defined goals.

So, just as the interfaces we design must be suitable for their systems and users, the models we use to reason about these interactive systems must also be useable and useful to their users (designers, formal practitioners etc.). Rather than having an expectation that UI designers should throw away their usual, and necessarily 
informal, design processes in favour of a new formal approach, we are of the opinion that these should be incorporated into our approach rather than be replaced by it.

We start with the assumption that traditional, informal user-centred design practice has been used, as usual, to produce many artefacts relating to the "look and feel" of the system. These will include design ideations such as prototypes, scenarios, story-boards etc. and will be the result of collaboration between designers and end-users, as well as other stake-holders. Keeping this point in mind is very important when it comes to understanding the modelling techniques that we discuss in this chapter.

In the same way that we assume the interface has been developed using wellknown and appropriate design methodologies, we similarly assume that the design of the functional behaviour of the system has likewise followed a rigorous development process which leads to the development of formal artefacts such as a system specification or some other formal model of the system's behaviour. Given that we are interested in safety-critical systems, where erroneous behaviour can lead to injury or even death, this assumption seems a reasonable one.

In order to be able to reason about all parts of the system and not just the functional behaviour, we need a way of considering the informal design artefacts with the same level of formality as the functional specification. To do this we create formal models of these design artefacts. By creating a formal representation of the informal design artefacts (in essence creating a different abstraction of the UI which happens to be in a formal notation) we gain the ability to combine the two representations (system and UI) into another model which gives a single view of the system as a whole. This then allows us to reason about the interactive system in a way which captures all of the information (both UI and system) and perform activities such as model-checking and theorem-proving to show that all parts of the system will have the properties we desire (Bowen and Reeves, 2008, 2013).

We next give an overview of the different models used in our process and then go on to provide examples of these in use for the nuclear power plant example.

\section{Background}

Our starting point for the modelling process is to consider the two main components of the system (functionality and interactivity - which includes the interface) separately. This separation of concerns is, in some sense, an artificial division. While it is often the case that one group within a design team may focus on appearance and look and feel of the interface while another focusses on core functionality we are not aiming to reflect the divisions and complexities that exist within design groups. Rather, the separation allows us to consider different parts of the system in different, and appropriate, ways, and provides the basis for our use of different levels of abstraction to describe different components within that system. Such separation is a common approach taken in this type of modelling although it can be done at differing levels of granularity. For example in chap. 5 we see functional behaviours 
described as components which are then associated with related widgets and composed using channels, whereas chap. 14 uses a layered approach where different parts of the system and model are described in different layers.

We rely on a combination of existing languages and models to specify the functionality. Typically, for us, this means creating a Z specification (ISO/IEC 13568, 2002; Henson et al, 2008) and/or $\mu$ charts (Reeve, 2005) to reason about functional and reactive behaviours, although any similar state-based notation could be substituted for Z. The ProZ component of the ProB tool ${ }^{1}$ is used for model-checking the specification, or we can use $Z$ theorem provers such as Proofpower ${ }^{2}$. These allow us to ensure that the system not only does the right thing (required behaviour) but also does not do the wrong thing (behaviour that is ruled out) irrespective of the circumstances of use. Figure 1 gives an overview of how each of the models relates to the system under consideration.

The presentation model describes the user interface elements that are present but not the layout of the interface. It also describes the types of interaction each widget exhibits (which suggests how a user interacts) and labels the behaviour associated with that widget. The presentation interaction model (PIM) also describes the user interface but at a higher level of abstraction which hides the general behaviours and widgets of the interface and focusses on the navigation that is possible through the different modes/windows etc.

The presentation model relation (PMR) relates some of the labels of widget behaviours to operations in the specification, and as such gives a very high level view of the interaction between system and UI. Not all functionality is expressed via the user interface (the user can only directly access certain behaviours) hence the split in the system functionality box where the grey section are those functions which do relate to user actions.

$\mu$ Charts is used as an alternative notation for PIMs, and so describes the same properties of the user interface, but can also be used to describe aspects of system functionality to show cause and effect behaviours. The specification on the other hand describes only the system functionality in terms of what can be achieved and abstracts away all detail of how it might be achieved.

Finally the models can be combined into single representation which gives a low level description of the what and how of the interface and system and their interaction. We do not explicitly model the user at all in our approach nor any backend processes such as database connectivity, networks etc. In the next section we describe each of the models in more detail using examples from the Nuclear Power Plant example.

\footnotetext{
${ }^{1}$ http://stups.hhu.de/ProB/

${ }^{2}$ http://www.lemma-one.com/ProofPower/index/index.html
} 


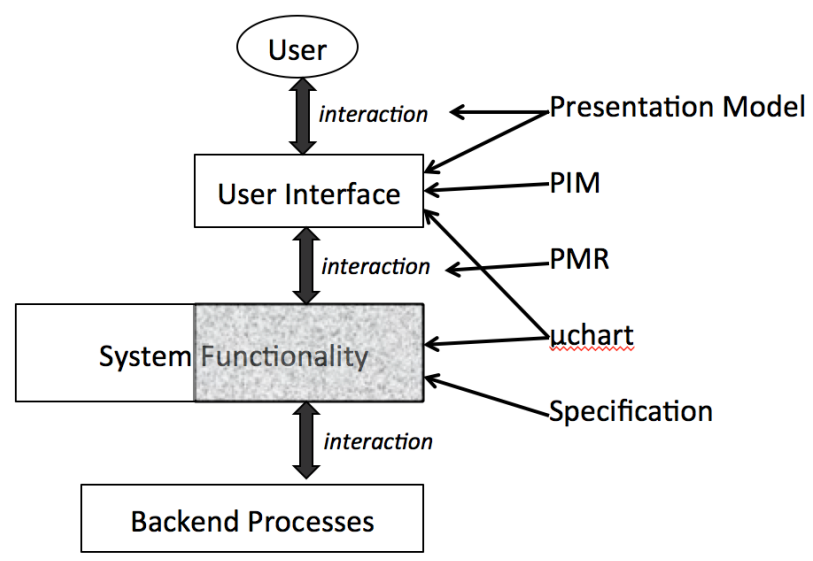

Fig. 1 Overview of model components

\subsection{Presentation Model}

This is a behavioural model of the interface of a system described at the level of interactive components (widgets), their types and behaviours. We group the widgets based on which components of the interface (e.g. windows, dialogs) they appear in (or in the case of modal systems, which modes they are enabled in). The presentation model can be derived from early designs of interfaces (such as prototypes, story-boards etc.), final implementations, or anything in between. As such they can be produced from the sorts of artefacts interface designers are already working with within a user-centred design process. The presentation model does not in any sense replace the design artefacts it describes. Rather, we use it as an accompanying artefact that provides a bridge between informal designs and formal models. So the presentation model describes the 'meaning' of the interface (or interface design) in terms of its component widgets and behaviours, but the layout and aesthetic attributes are contained in the visual artefacts that the presentation model is derived from.

It is important to appreciate that we do not require widgets to be (only) buttons or check boxes or menu items etc. Widgets are any 'devices' through which interaction can occur. For example, sensors attached to parts of a physical system would be widgets: as the physical system evolves the sensors would note this evolution and, in response, the system would move between states. In this sort of system we might simply describe a collection of sensors together with the behaviours (both functional and interactive) that their triggering or their readings cause in the system. Thus our idea of 'interface' is very general. 
Each window, dialogue, mode or other interactive state of the system is described separately in a component presentation model (pmodel) by way of its component widgets which are described using a triple:

widget name, widget type, (behaviours)

The full interface presentation model is then simply the collection of the pmod$e l s$, and describes all behaviours of the interface and which widgets provide the behaviours. Behaviours are split into two categories, interactive behaviours (Ibehaviours) are those which facilitate navigation through the interface (opening and closing new windows, noting the change of a sensor state etc.) or affect only presentational elements of the interface, whereas system behaviours (S-behaviours) provide access to the underlying functionality of the system (the grey part in figure $1)$.

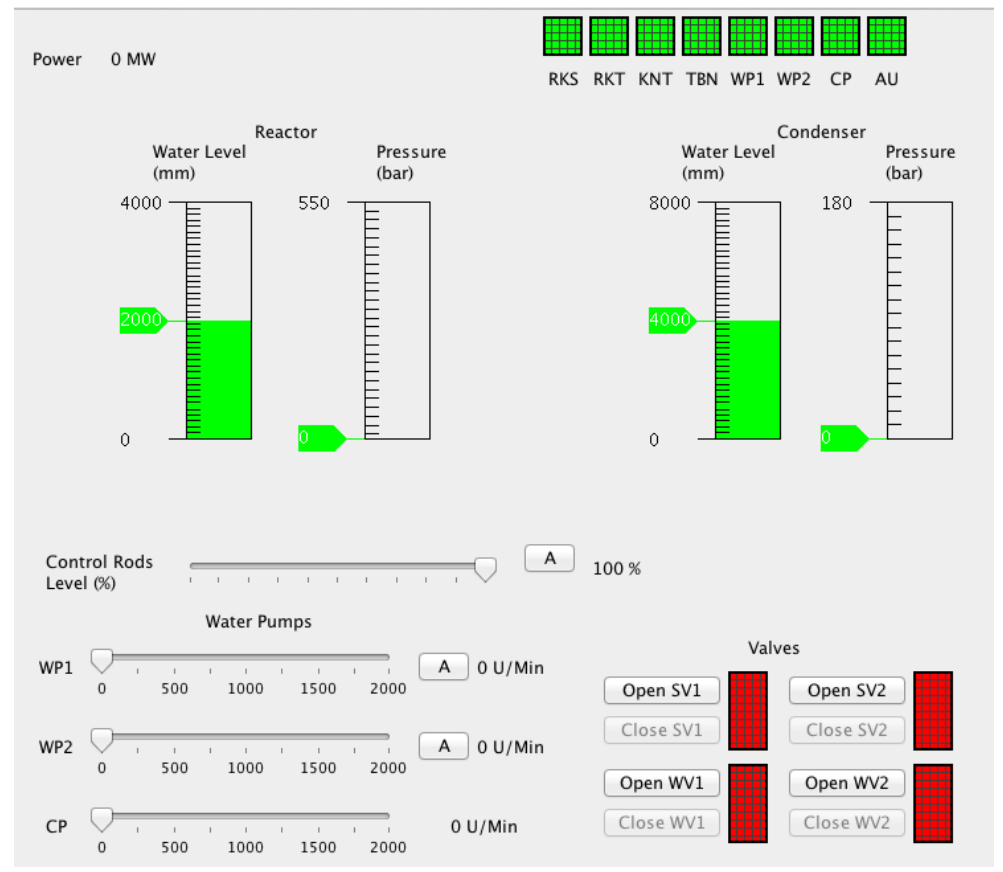

Fig. 2 Nuclear plant control example interface

The syntax of the presentation model is essentially a set of labels which we use to meaningfully describe the attributes of the widgets. Consider the interface provided as part of the nuclear power plant example, which we repeat in figure 2. Each of the widgets is described in the manner described, so for example the power output label in the top left corner is:

PowerDisplay, Responder, (S_OutputPower) 
The category assigned is that of 'Responder' as this widget displays information to the user in response to some inner stored value (which keeps track of the current power level). As such the behaviour it responds to is a system behaviour which outputs whatever that power level currently is, and hence has the label 'S_OutputPower'. The WP1 slider on the other hand is described as:

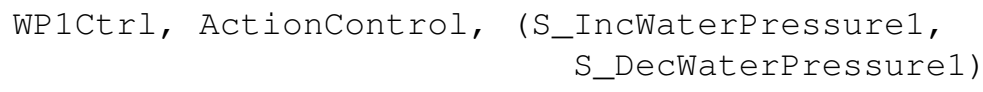

The category 'ActionControl' indicates it is a widget which causes an action to occur (i.e. the user interacts with it to make something happen), which in this case is to change the value of the water pressure either up or down. Again these are behaviours of the system and so are labelled as S-behaviours.

Once we have labelled all of the widgets in the UI we have an overview of all the available behaviours that are presented to a user by that UI. We subsequently give a formal meaning to these labels via the presentation interaction model (PIM) and presentation model relation (PMR) which we describe next.

\subsection{Presentation Interaction Model}

The presentation interaction model (PIM) is essentially a state transition diagram where pmodels are abstracted into states and transitions are labelled with I-behaviours from those pmodels. As such the PIM gives a formal meaning to the I-behaviours as well as providing an abstract transition model of the system's navigational possibilities. The usual 'state explosion' problem associated with using transition systems or finite state to model interactive systems is removed by the abstraction of pmodels into states, so the size of the model is bounded by the number of individual windows or modes of the system. While the presentation model describes all available behaviours the PIM describes the availability of these via the user's navigation. For example in a UI with multiple windows the user may be required to navigate through several of these windows to reach a particular behaviour. The nuclear power plant example of figure 2 has only a single, static UI screen and as such the PIM is a single state automaton. We show later how this changes when we extend the example to have multiple windows constraining behaviour in the case of the emergency scenarios.

A PIM can also be used to consider aspects such as reachability, deadlock and the complexity of the navigational space (via the lengths of navigational sequences). Considered formally, the role of the PIM is to inform us what the allowable sequences of $\mathrm{Z}$ operations are for the interactive system that we are modelling. This allows us to make the $Z$ definitions somewhat simpler since we do not have to introduce an elaborate system of flags and consequent pre-conditions in order to disallow the use of $Z$ operations when the interactivity does not allow them: the PIM handles all of this, and what $Z$ operations are allowed at any point in the interactivity is given by what widgets have behaviours (that are given by the $\mathrm{Z}$ operations) at that point. 


\subsection{Presentation Model Relation}

Just as the PIM gives meaning to the I-behaviours of the presentation model, the presentation model relation (PMR) does the same for the S-behaviours. These behaviours represent functional behaviours of the system, which are specified in the formal specification. The PMR is a many-to-one relation from all of the Sbehaviours in a presentation model to operations in the specification. This reflects the fact that there are often multiple ways for a user to perform a task from the UI and therefore there may be several different S-behaviours which relate to a single operation of the specification.

So, in order to understand what an S-behaviour label represents, for example the behaviour label $S$ IncWaterPressurel from the presentation model tuple above, we identify from the PMR the name of the operation in the $\mathrm{Z}$ specification that it represents:

\section{S_IncWaterPressure $1 \mapsto$ IncreaseWaterPressure}

This tells us that in the formal specification is an operation called 'IncreaseWaterPressure' which specifies what effect this operation has on the system. The specified operation then gives the meaning to this behaviour label. We give a larger example of the PMR for the nuclear power plant example later.

\subsection{Specification}

The formal specification of the system provides an unambiguous description of the state of the system and the allowable changes to that state provided by the operations. Many different formal languages exist for such a specification (e.g. VDM, Z, B, Event-B, Object-Z etc. to name but a few) but for our approach we rely on the $\mathrm{Z}$ specification language which is based on set theory and first order predicate logic. In Z, we typically give a description of the system being modelled which is based on what can be observed of it, and then the operation descriptions show how (the values of) what is observed change. The operations are guarded by preconditions which tell us under what circumstances they are allowed to occur (i.e. based on given values of observations or inputs) and the postcondition defines which observations change and which do not when the operation occurs as well as describing any output values.

The specification of the nuclear power plant example, therefore, is concerned with observations relating to the items such as reactor pressure and water levels, condenser pressure and water levels, power output, speed of the pumps, status of the valves etc. A Z specification can be used with theorem provers to prove properties over the entire state space of the system (for example to show that a certain set of observations is never possible if it describes an undesirable condition) and can also be used with model-checkers to examine the constrained state space for things such as safety conditions. 


\section{$3.5 \mu$ Charts}

In addition to the $\mathrm{Z}$ specification, we also use the visual language, $\mu$ Charts (Reeve, 2005; Reeve and Reeves, 2000b,a) (a language used to model reactive systems). PIMs can also be represented as $\mu$ charts, which provides additional benefits over a simple PIM (including the ability to compose specific sets of behaviours in different charts via a feedback mechanism and embed complex charts into simple states in order to 'hide' complexity) (Bowen and Reeves, 2006a).

$\mu$ Charts is based on Harel Statecharts (Harel, 1987) and were developed by Philipps and Scholz (Scholz, 1996; Philipps and Scholz, 1998). $\mu$ Charts was subsequently extended by Reeve (Reeve, 2005) and we use his syntax, semantics and refinement theory. $\mu$ Charts has a simpler syntax than Statecharts (and in some sense can be considered a 'cut down' version) and it also has a formal semantics. $\mu$ Charts and Statecharts differ in terms of synchrony of transitions (we imagine a clock ticking and a step happening instantaneously at each tick), step semantics and the nature of the labels on transitions. Labels on transitions in $\mu$ charts (note we refer to the language as $\mu$ Charts and the visual representations as $\mu$ charts) are of the form guard/action where guards are predicates that trigger a transition if they are true, and also cause actions to take place. For example, if a guard is simply a signal $s$ then the presence of $s$ in the current step makes the guard true (think of the guard as being the predicate "the signal $s$ is present"). An example of an action is "emit the signal $t$ in the current step". Guards are evaluated and actions happen instantaneously in the same single step, thus the emission of a signal from one transition which is a guard to another transition results in both transitions occurring in the same step.

The $\mu$ Charts language includes several refinement theories which in turn gives us refinement theories for PIMs. The trace refinement theory for $\mu$ Charts is particularly useful as it can be abstracted into a much more lightweight refinement theory for interfaces based on contractual utility (Bowen and Reeves, 2006b). The semantics of $\mu$ Charts is given in $\mathrm{Z}$ and there is a direct translation available (via an algorithm and tool) from a $\mu$ chart to a $Z$ specification (Reeve and Reeves, 2000b), this in turn means we have an algorithm and means to turn a PIM into a $\mathrm{Z}$ specification (Bowen and Reeves, 2014).

\subsection{Combining the Models}

The models of functionality (specification) and interactivity (presentation model and PIM) are already coupled via the PMR. This gives us a model which combines the conventional use of $\mathrm{Z}$ to specify functionality together with the more visually appealing use of charts for the interactivity.

However, we can also combine the models in a way that leads to a single model, all in $\mathrm{Z}$, of the entire system. This gives us the ability to, for example, create a single model of all parts of an interactive system (i.e. interactivity and underlying functionality), that can then be used to prove safety properties about that system 
which might relate to functional constraints, interface constraints, or both (Bowen and Reeves, 2013). It might also be used as the basis for refinement, ultimately into an implementation, via the usual refinement theories for $\mathrm{Z}$ (Derrick and Boiten, 2014; Woodcock and Davies, 1996).

We do this single-model building by using the $\mathrm{Z}$ semantics of $\mu$ Charts and by expressing the PMR as a $\mathrm{Z}$ relation. This is then combined with the formal specification of the functionality of the system, giving a single model where the transitions representing the PIM are used to constrain the availability of the operations. So if an S-behaviour given in the presentation model is only available in one state of the $\mathrm{UI}$, this is represented in the new combined $\mathrm{Z}$ specification as a pre-condition on the related operation. Recently we have also given a simplified semantics for the creation of a single specification from the models to reflect models of modal devices where the PIMs typically do not use the full expressiveness of $\mu$ Charts (Bowen and Reeves, 2014).

\section{The Nuclear Power Plant Case Study}

We now consider the case study and give concrete examples of the modelling techniques described above. From the general overview of the functionality of the nuclear power plant given in the case study (along with several assumptions to fill in the gaps) we can generate a $\mathrm{Z}$ specification of the desired functionality. This $\mathrm{Z}$ specification gives us a description of the observable states of the system (i.e. its state space) as a $\mathrm{Z}$ state schema, along with the operations that can change these states (i.e. move us around the state space). As such, it formally specifies behaviours that can be exhibited by the system. ${ }^{3}$ Note that we have simplified the value types for pressure and water levels to natural numbers for convenience of specification.

The observable states of the system can be captured by observing all of the parameters that are described in the case study brief along with any known constraints. For example, we know that for the state of the reactor to be considered 'safe' then the reactor pressure must be no more than 70 bar while the core temperature remains below the maximum value of $286^{\circ} \mathrm{C}$. So, we would have to be able to observe these values, and ensure that they are within the required limits, in order to be able to say that we are describing an allowed state of the system.

The state space of the system is given by a state schema like ReactorControl below. It declares names and types for all the observations that can be made of parts (or parameters) of the system, and it places constraints on some or all of those observations in order to ensure that the state space contains only safe states. Of course, this is a design decision; it is equally valid to have a state space that includes unsafe values for some observations, as long as this aspect is handled elsewhere in the specification (e.g. perhaps there are specialised error-handling operations that

\footnotetext{
${ }^{3}$ Of course in order to ensure this is actually true we must also consider the preservation of these properties in the final implementation, but we will not go into a discussion about refinement here.
} 
come into play once we enter a state which is allowed by the model but which is unsafe according to the requirements).

The $\mathrm{Z}$ snippets below show some of the definitions used to model the system.
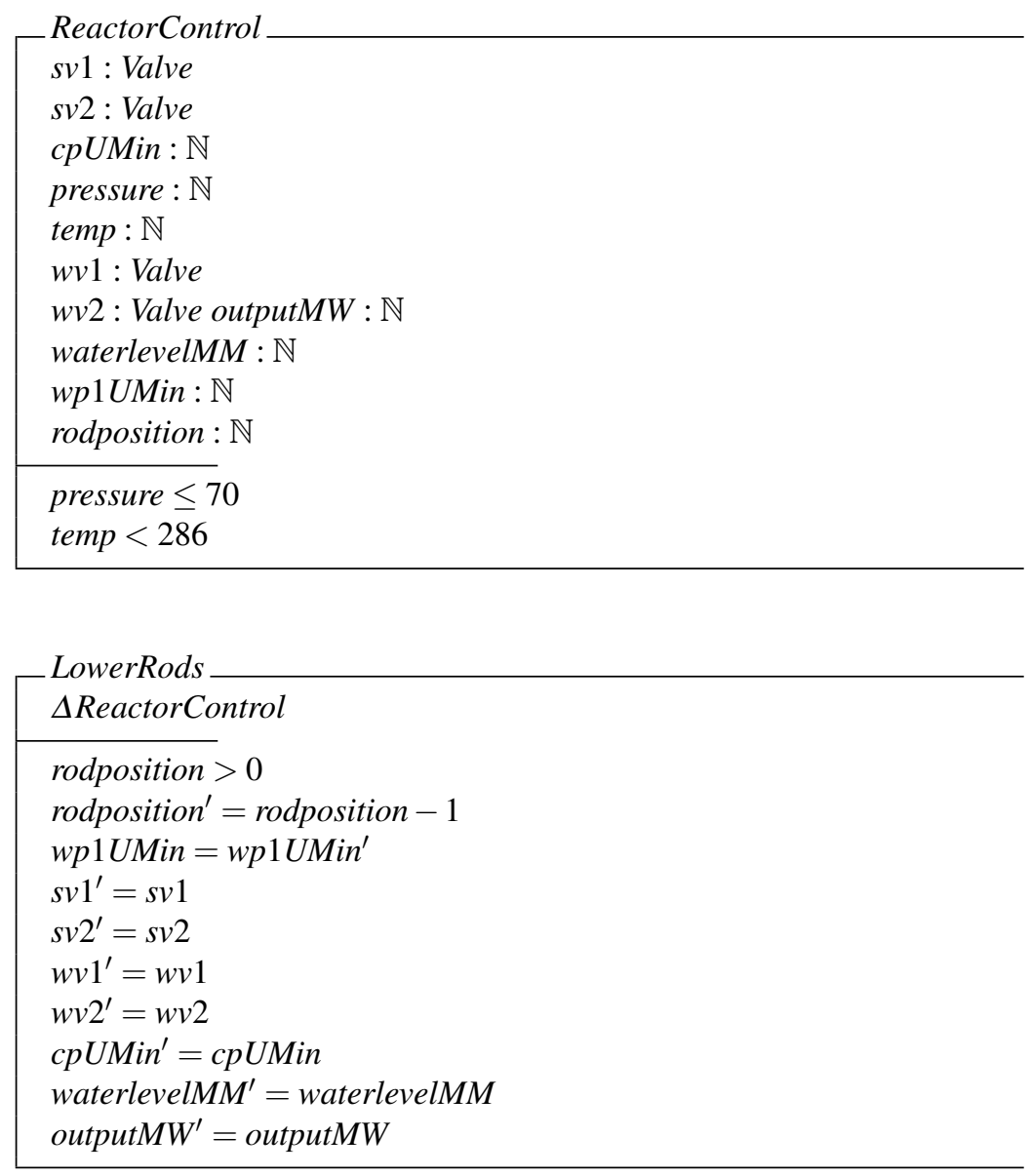

The ReactorControl schema is the state schema showing the observable states of the system (via the parameters or observable values within the system) and LowerRods is an operation schema that changes a state - in this case the position of the rods is the only part of the overall state that changes.

Once we have a complete specification of all allowable behaviours we can then use a model-checker such as the ProZ plug-in for ProB to investigate the specification to ensure it behaves as expected. For example, if we have specified what it means for the system to be in a stable state then we should be able to show that when these conditions are not satisfied then the system moves into one of the three error control states - abnormal operation, accident or SCRAM. 


\begin{tabular}{l} 
Stable \\
EReactorControl \\
\hline $\begin{array}{l}\text { cpUMin }=1600 \\
\text { waterlevelMM }=2100 \\
\text { outputMW }=700\end{array}$
\end{tabular}

\section{Status $\widehat{=}$ Stable $\vee$ Abnormal $\vee$ Accident $\vee S C R A M$}

In the same way as we describe the system observations for Stable we also describe the Abnormal, Accident and SCRAM states so that Status is then defined as the disjunction of these possible states.

While this high-level view is essential in enabling us to perform the sorts of proofs we require for a safety-critical system we may wish to consider subsets of behaviour in more detail. We could always, of course, add to the specification to include all of the detail needed to consider these, but it is true that the more we reduce the level of abstraction the more unreadable (and potentially unwieldy) the specification becomes.

Suppose we wish to consider some specific procedures of the power plant which combine several operations. For example, when the plant starts up or shuts down there are a series of required steps that must occur along with requirements of values of certain parameters (i.e. conditions on observable values) that enable the required steps to proceed. We could investigate the 'Start-up' and 'Shut-Down' steps using model-checking with the $\mathrm{Z}$ specification as above, however in order to more easily consider the user inputs to control these procedures (which are not included in the specification) we might instead create a $\mu$ chart which shows the required input levels and reactions that occur in these processes.

Figure 3 shows the $\mu$ chart of the 'Start-up' procedure for the power plant. It provides a different (and more visual) abstraction of the system than the $\mathrm{Z}$ specification might be able to and is more expressive in terms of the reactive properties (the $\mathrm{Z}$ is intended to describe what is and is not possible and abstracts away such reactive and event-driven behaviour deliberately), but at the same time (via its $\mathrm{Z}$ semantics) retains all of the useful properties of a formal specification. In particular the $Z$ specification, while it tells us precisely what the state space is and what operations can move us around the state space, says nothing about the sequencing of operations that are allowed or possible. This is, in part, the role of the $\mu$ chart. For example, the fact that a valve must be open before it can be closed is best handled by the chart. It could formally be handled via setting-up flags and preconditions for $\mathrm{Z}$ operations in terms of those flags, but this is an example of the unwieldiness of one language over another: picking the right language for each part of a job is part of the skill of a modeller, and part of the elegance that comes from experience. The fact that the modelling could all be done in Z, say, is no reason to actually do it all in Z. If there is a better language for certain parts of the model, in this case the use of a chart for 
the sequencing of operations, then the elegant solution is to do the modelling in that way.

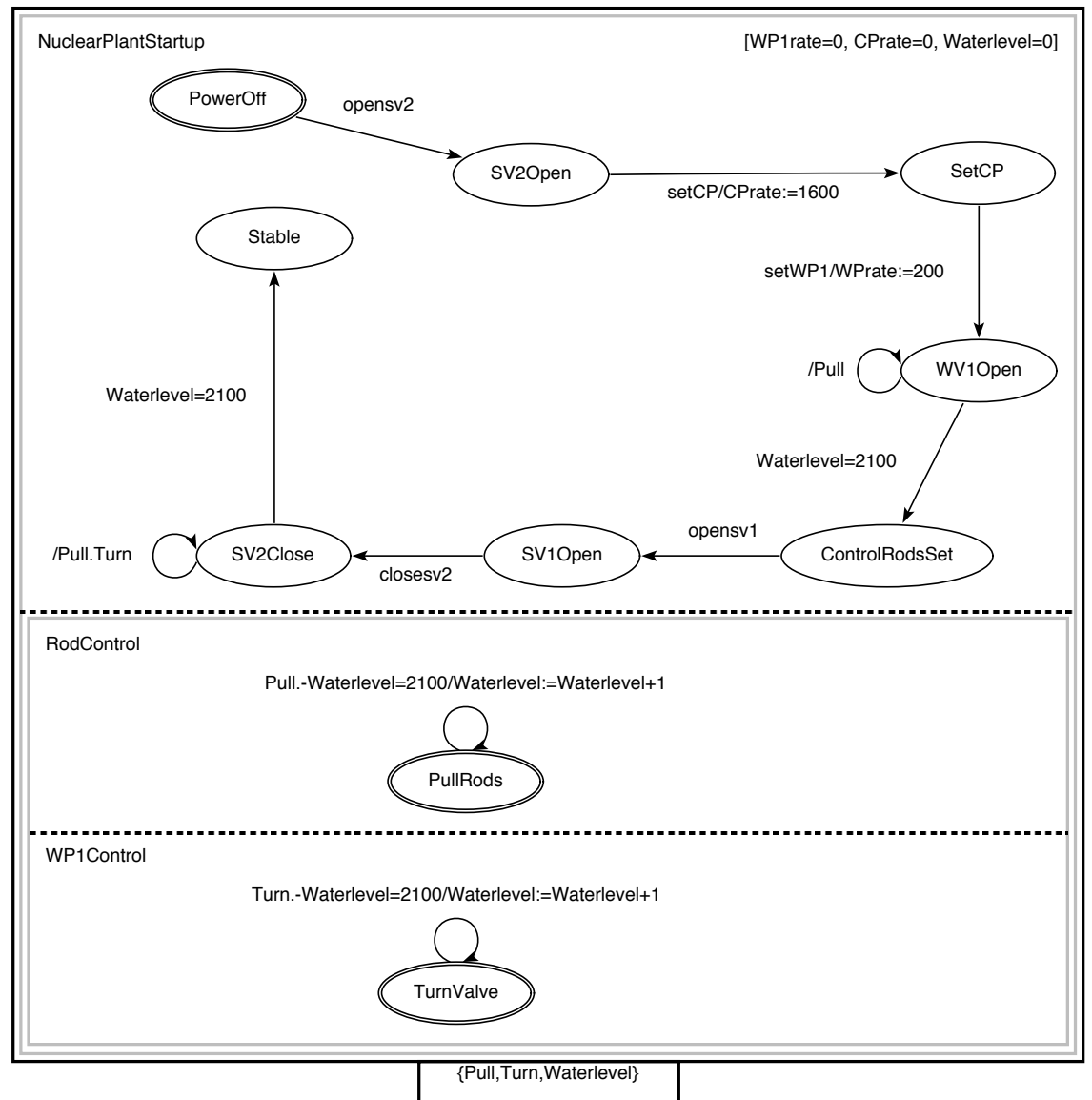

Fig. 3 Chart of startup procedure

The model consists of three atomic $\mu$ charts (which are in this case just like simple finite-state machines) composed in parallel, which means that they each react in step with each other and signals listed in the feedback box at the bottom of the chart are shared instantaneously between all charts at each step. This modularity is another of the advantages of using $\mu$ Charts as we can explicitly model relationships between independent components and behaviours. For example in this chart we can see that once the system is in the svclose 2 state, it will remain there until the water level reaches the required value, and at each step it outputs signals (from the self-loop 
transition) Pull and Turn ${ }^{4}$. These signals are then shared with the RodControl and WPIControl charts which lead to transitions which ultimately affect the water level until the required value is reached.

The transitions between the various states the system goes through are guarded by required values on key indicators (such as water level, power output etc.) as well as user operations (such as opening and closing valves). In this model we still do not distinguish between user operations, system controlled operations and functional monitoring of values. In this way we still abstract from a user's view of the system as we are most interested here in ensuring the correct outcomes are reached depending on the values and that the components interact properly as shown by the feedback mechanism of the composed charts.

Using the $\mathrm{Z}$ semantics of $\mu$ Charts, and a tool called ZooM ${ }^{5}$ which generates the $\mathrm{Z}$ specification which expresses the meaning of a $\mu$ chart, we can go on to modelcheck this component of behaviour to ensure that the system progresses correctly through the start-up procedure (and similarly shut-down) only when the correct pre/post conditions are met. Already having these two different, but inter-related, views gives us a consistent mechanism for viewing parts of the system in different ways.

Once we are satisfied that the system will behave correctly as described we must also ensure that the users can perform the required operations and that at the very least the interface provides the necessary controls (we do not talk about the issue of usability of the interface in this chapter-recall our comment in the introduction about the usual artefacts being available from the UCD process-however it is of course equally important in ensuring the system can be used): so we take any design artefacts we have for the user interface to the control system and create presentation models, PIMs and PMR as described previously.

For the nuclear power plant control system we start with the initial design shown in figure 2. The following presentation model and PMR snippet gives an example of the models derived from this. There is no PIM at this stage as we are dealing with a single fixed 'window' which has no navigational opportunities for the user and so, as mentioned previously, it is trivially a single-state automata.

\section{Presentation Model}

PowerDisplay, Responder, (S_OutputPower),

RWaterLevelDisplay, Responder, (S_OutputReactorWaterLevel),

RPressureDisplay, Responder, (S_OutputReactorPressure),

ControlRodCtrl, ActionControl, (S_RaiseControlRods, S_LowerControlRods),

WP1Ctrl, ActionControl, (S_IncWaterPressure1, S_DecWaterPressure1),

WP2Ctrl, ActionControl, (S_IncWaterPressure2,S_DecWaterPressure2),

\footnotetext{
${ }^{4}$ Note that these two transitions happen at every clock tick since their guards are true, denoted by convention by the absence of any guard.

${ }^{5} \mathrm{http} / / /$ sourceforge.net/projects/pims1/files/?source=directory
} 
CPCtrl, ActionControl, (S_IncCPressure, S_DecCPressure),

SV1Open, ActionControl, (S_OpenSV1),

SV1Close, ActionControl, (S_CloseSV1)

SV1Status, Responder, (S_OutputSV1Status)

PMR

S_OutputPower $\mapsto$ OutputPowerLevel

S_OutputReactorWaterLevel $\mapsto$ OutputReactorWaterLevel

S_OutputReactorPressure $\mapsto$ OutputReactorPressure

S_RaiseControlRods $\mapsto$ RaiseRods

S_LowerControlRods $\mapsto$ LowerRods

S_IncWaterPressure $1 \mapsto$ IncreaseWaterPressure

S_DecWaterPressure $1 \mapsto$ DecreaseWaterPressure

S_OpenSV1 $\mapsto$ OpenSV1

S_CloseSV1 $\mapsto$ CloseSV1

S_OutputSV1Status $\mapsto$ OutputSV1Status

For brevity we do not include all of the status lights and valve controls (e.g. for valves SV2, WV1 and WV2) but the reader can assume they are described in the same manner as the SV1 controls and status display.

The presentation model can be used to ensure that all of the required operations are supported by the user interface, whilst the PMR ensures that the UI designs are consistent and complete with respect to the functionality of the system. For example, if we have some S-behaviours of the presentation model which do not appear in the PMR then we know that the interface describes functionality that is not included in the specification and we must therefore address this incompleteness.

We can also use these interface models to help derive alternative (restricted) interfaces for use in error conditions when the user may have only partial control of the system, or when they have no control due to SCRAM mode. Initially a presentation model of the alternative interfaces provides information about what operations are (and more crucially, are not) available for the user. Subsequently we can use the refinement theory based on $\mu$ Charts trace refinement (Bowen and Reeves, 2006b) to examine alternatives and prove that they are satisfactory. The visual appearance of the alternative interfaces may be entirely different from the original (although of course we would want as much correspondence between interfaces for the same system as possible to avoid user confusion). The presentation models of the different interfaces allow us to compare behaviours (via the refinement theory) irrespective of the appearances.

The interface in figure 2 allows the user full control of all aspects of the system, as occurs when it is in a stable mode. However, if it moves into one of its error states then the user has a reduced amount of control (or none in SCRAM mode when the system functions in a totally automated fashion). We might propose changes to the interface to support this restricted control and provide feedback to the user about what is happening and what they can, and cannot, do. Figure 4 shows a suggested design change to the nuclear power plant for when the control system is in 'Abnormal' mode, and partial automated-only control is in place. 


\begin{tabular}{|c|c|c|c|c|c|}
\hline & \multicolumn{5}{|c|}{ Pumps under automatic control } \\
\hline & 0 & 500 & 1000 & 1500 & 2000 \\
\hline WP2 & 0 & 500 & 1000 & 1500 & 2000 \\
\hline $\mathrm{CP}$ & 0 & 500 & 1000 & 1500 & 2000 \\
\hline
\end{tabular}

Fig. 4 Restricted interface for pump controls

The presentation model for the interface will then differ from that of the original example as the three pump controls are now displays rather than controls (they show the user what the automated system is doing rather than enable the user to make changes themselves). The widgets are still associated with the same behaviours, but instead of generating these behaviours (as action controls do) they now respond to them instead. In addition, we include the automated behaviour (which drives the mode switch), described as a 'SystemControl', which leads to the interface behaviours of changing the display.

WP1Ctrl, Responder, (S_IncWaterPressure1,S_DecWaterPressure1)

WP2Ctrl, Responder, (S_IncWaterPressure2,S_DecWaterPressure2)

CPCtrl, Responder, (S_IncCPressure, S_DecCPressure)

Status, SystemControl, (S_Stable, I_Stabilised)

Similarly we add the automated behaviour to the original presentation model to make explicit the automation which switches into abnormal mode.

Status, SystemControl, (S_Abnormal, I_AbnormalOperation)

It is important to ensure that this new interface provides exactly the right controls and restrictions to the user, and also that they are only present in the relevant error states (i.e. that a user is not restricted when the system is stable).

For each of the possible error states, Abnormal, Accident and SCRAM we can provide different interfaces which provide only the correct levels of user interaction. Figure 5 shows the PIM for the new collection of interfaces, including those of the other error modes (Accident and SCRAM) although we do not discuss their designs here.

The Stable state is considered the initial state (indicated by the double ellipse) and the transitions indicate possible movements between states. The SCRAM state is a deadlock state, in that there are no possible transitions out of this state. This is 


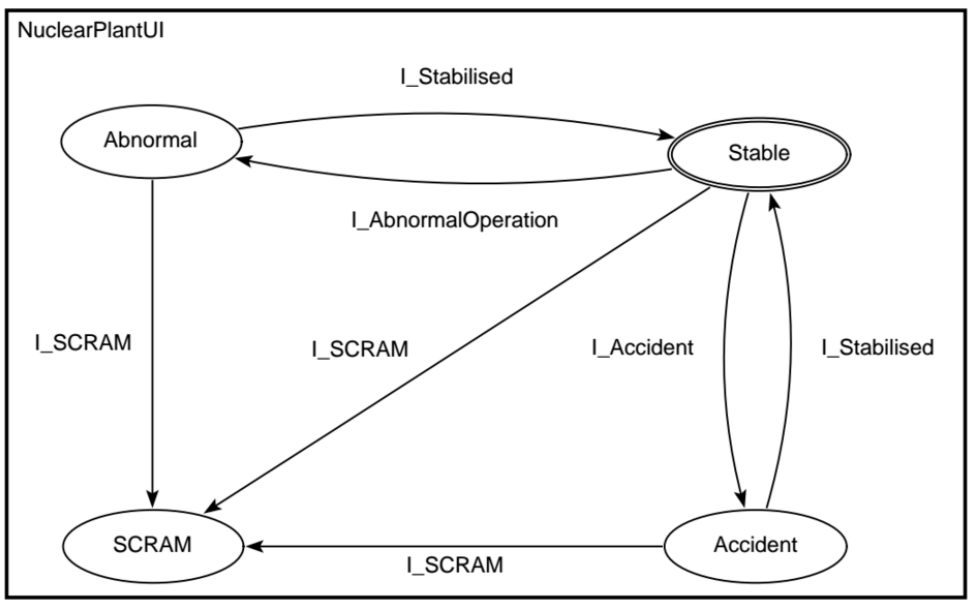

Fig. 5 PIM of interface modes

correct for this system as the user cannot interact with the system in this mode and the only possible behaviour for the system is to go into a safe shutdown mode.

There are several types of properties we may wish to consider once we have these designs and their associated models. First, we should ensure that the combination of new interface models still adhere to the original requirements. Second, as stated above, we should be sure that the correct level of control/restriction is provided in each instance.

Typically when we make changes to the interface or interaction possibilities of our system during the modelling stage we would use refinement to ensure the adherence to original requirements. However, what we are doing with the new interfaces is restrict behaviour, so it is not the case that each of the different (new) modes refines the original, but rather that the total interface model (the concatenation of the four pmodels) refines the original. We reiterate that we retain all of the visual designs of layout etc. for both the original as well as the new UIs so that we can always refer back to these to understand more about the actual appearance. This will, of course, be crucial when we come to evaluate the usability aspects of the UIs. In terms of the final considerations of refinement, however, we rely on the models alone.

Refinement for interface and interaction properties described in presentation models and PIMs is based on the notion of contractual utility (Bowen and Reeves, 2006b) and can be described by relations on the sets of behaviours of the models in a simple way, whilst being formally underpinned by the trace refinement theory of $\mu$ Charts. Given two arbitrary interfaces, $A$ and $C$, The requirements for the two types of behaviours are as follows:

$\mathrm{UI}_{A} \equiv_{S B e h} \mathrm{UI}_{C}$

$\mathrm{I} \_\mathrm{Beh}\left[\mathrm{UI} \mathrm{I}_{A}\right] \subseteq \mathrm{I} \_\mathrm{Beh}\left[\mathrm{UI}_{C}\right]$ 
where $\mathrm{I} \_\mathrm{Beh}[\mathrm{P}]$ is a syntactic function that returns identifiers for all I-behaviours in $\mathrm{P}$.

We call the first interface (from figure 2) 'Original' and the new interfaces (a combination of 'Original' with the addition of the automation and 'Abnormal') 'New'. We use the syntactic functions to extract behaviours to create the following sets:

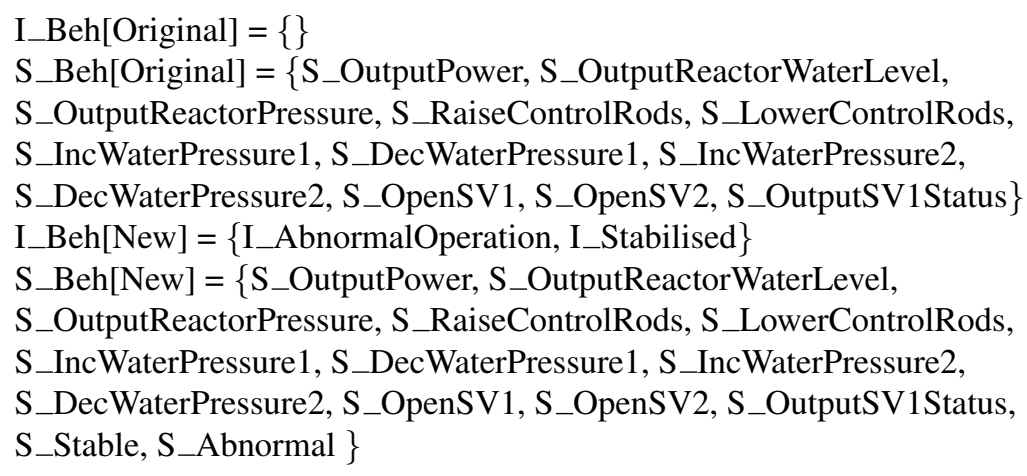

The requirement on the I-behaviours permits addition of new behaviours, and so this is satisfied. However, notice the inclusion of the S-behaviours for the automation to switch the system between states of the interface (S_Stable and S_Abnormal). These are now implicit behaviours of the interface and so must also be included, but these additional behaviours break the requirement on equality between sets of S-behaviours of the interfaces. If we consider this further we can understand why this is a requirement. Our new interface depends on behaviours to switch between modes under different states of the system which were not part of the original description, as such there is no guarantee that this behaviour is described anywhere in the specification. We can see from the PMR that there is no relation between these operations and the specification, so we have added functionality to the interface may not (yet) be supported by system functionality. This is an indication that we need to also increase behaviours in the functional specification which will allow these to then be supported, or we need to ensure that we can relate any existing specified operations (via the PMR) to the new S-behaviours. In fact we have already discussed earlier how we can describe the different states of the system (Stable, Abnormal, SCRAM etc.) in the specification so we must now ensure that identification of these states along with the necessary behaviour is also described.

Now we can investigate the behaviours of the component pmodels (of each different mode of use) via translation of the PIM (to Z) and its corresponding specification to ensure that these correspond to permissible user behaviours in each of the system states. We discuss the combination of models which provides this next. 


\subsection{Benefits of Combining the Models}

In the previous sections we have given examples of the use of the individual models to consider some properties of the nuclear power plant control system which might be of interest during the development process. There are some things, however, which require a combined model of both UI and functionality in order to formally consider. Suppose we want to ensure that when the system is in Abnormal mode, the user cannot alter the water pressure (as this is one of the elements under automatic control in this mode). Using the ProB model checker there are several different ways to perform such analysis, and we have found that describing the properties as LTL formulae and then checking these is a useful mechanism as ProB provides counterexamples consisting of a history list of operations performed when a formula check fails (Bowen and Reeves, 2014).

However, the functional specification alone cannot be used for this. If we perform some analysis to show that when the status of the system is Abnormal the operations to increase or decrease the water pressure are not enabled, we find that this is not true. Of course, this is exactly as it should be, although the user cannot change the water pressure, it can still be changed (via automation) and our functional model correctly describes this.

In order to consider the possible effects of user interactions, therefore, we need to combine the interface models with the specification. We start by declaring types for the states of the PIM and the transition labels.

State $::=$ Stable $\mid$ Abnormal $\mid$ Accident $\mid$ SCRAM

Signal $::=I \_$AbnormalOperation $\mid$I_Accident $\mid$I_SCRAM $\mid$I_Stabilised

Next we give a description of the UI which consists of a single observation which shows the state the UI is in (one of the states of the PIM) and create an operation schema for each of the transitions which describes the change in state that occurs on a given signal, for example:

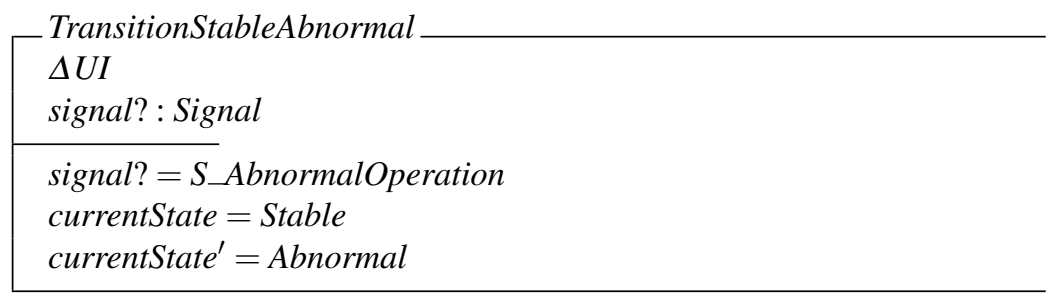

This describes how the observation of 'currentState' changes when the transition from 'stable' to abnormal' occurs, which requires the 'I_AbnormalOperation' input signal to be present.

The final step is to create a combined schema for the system and UI (so we include the schema descriptions for each into a single schema) and then for each of the operation schemas we add a precondition which gives the required state of the 
UI, i.e. shows when the operation is available to a user. Now when we model-check the specification and check the condition of whether a user can change the water pressure when the system is in an abnormal state we find that they cannot, as none of the user operations which change the water pressure are enabled if the system state is abnormal.

In order to create a fuller picture we should include all of the automation considerations. We can do this by describing automatic control in exactly the same way as we have shown above. That is, we create the alternate set of models including automated interface behaviours (as if they were user controls) and then we can include this with the combined model. This new model then enables us to show that the correct levels of manual (human user) or automatic control occur in all of the different states of the system.

\section{Conclusion}

In this chapter we have described the different models we use for reasoning about interactive systems. Using the nuclear power plant control system as an example we have shown how the models can be used independently as the differing expressive natures of the languages involved mean that they are individually suitable for different tasks in the process of verifying and validating safety-critical interactive systems. We have also given an example of combining the models into a single formal description, which allows us to ensure correctness of the interactivity and interaction in combination with the functionality.

Although the type of interface used in the nuclear power plant control example consists of standard desktop system controls (buttons, sliders etc.) this is not a requirement for our methods. Any type of interaction control (speech, touch, gesture, sensor etc.) can be modelled in the same way as we can abstract them in the presentation models as event generating (action controls) or responding (responders) as shown here.

\section{References}

Bolton ML, Bass EJ (2010) Formally verifying human-automation interaction as part of a system model: Limitations and tradeoffs. Innovations in Systems and Software Engineering: A NASA Journal 6(3):219-231

Bowen J, Reeves S (2006a) Formal models for informal GUI designs. In: 1st International Workshop on Formal Methods for Interactive Systems, Macau SAR China, 31 October 2006, Electronic Notes in Theoretical Computer Science, Elsevier, vol 183, pp 57-72

Bowen J, Reeves S (2006b) Formal refinement of informal GUI design artefacts. In: Australian Software Engineering Conference (ASWEC'06), IEEE, pp 221-230 
Bowen J, Reeves S (2008) Formal models for user interface design artefacts. Innovations in Systems and Software Engineering 4(2):125-141

Bowen J, Reeves S (2013) Modelling safety properties of interactive medical systems. In: 5th ACM SIGCHI Symposium on Engineering Interactive Computing Systems, ACM, EICS '13, pp 91-100

Bowen J, Reeves S (2014) A simplified Z semantics for presentation interaction models. In: FM 2014: Formal Methods - 19th International Symposium, Singapore, pp 148-162

Courtney A (2003) Functionally modeled user interfaces. In: Interactive Systems. Design, Specification, and Verification. 10th International Workshop DSV-IS 2003, Springer Verlag Lecture Notes in Computer Science LNCS, pp 107-123

Derrick J, Boiten E (2014) Refinement in Z and Object-Z: Foundations and Advanced Applications, 2nd edn. Formal Approaches to Computing and Information Technology, Springer

Dix A, Runciman C (1985) Abstract models of interactive systems. People and Computers: Designing the Interface pp 13-22

Duke DJ, Harrison MD (1995) Interaction and task requirements. In: Palanque P, Bastide R (eds) Eurographics Workshop on Design, Specification and Verification of Interactive System (DSV-IS'95), Springer-Verlag, pp 54-75

Duke DJ, Faconti GP, Harrison MD, Paternò F (1994) Unifying views of interactors. In: Advanced Visual Interfaces, pp 143-152

Duke DJ, Fields B, Harrison MD (1999) A case study in the specification and analysis of design alternatives for a user interface. Formal Asp Comput 11(2):107-131

Harel D (1987) Statecharts: A visual formalism for complex systems. Science of Computer Programming 8(3):231-274

Harrison MD, Dix A (1990) A state model of direct manipulation in interactive systems. In: Formal methods in human-computer interaction, Cambridge University Press, pp 129-151

Henson MC, Deutsch M, Reeves S (2008) Z Logic and Its Applications, Springer: Monographs in Theoretical Computer Science. An EATCS Series, pp 489-596

Hussey A, MacColl I, Carrington D (2000) Assessing usability from formal userinterface designs. Tech. Rep. TR00-15, Software Verification Research Centre, The University of Queensland

ISO/IEC 13568 (2002) Information Technology-Z Formal Specification Notation-Syntax, Type System and Semantics, 1st edn. Prentice-Hall International series in computer science, ISO/IEC

Jacob RJK (1982) Using formal specifications in the design of a human-computer interface. In: 1982 conference on Human factors in computing systems, ACM Press, pp 315-321

Limbourg Q, Vanderdonckt J, Michotte B, Bouillon L, López-Jaquero V (2004) UsiXML: A language supporting multi-path development of user interfaces. In: 9th IFIP Working Conf. on Engineering for Human-Computer Interaction jointly with 11th Int. Workshop on Design, Specification, and Verification of Interactive Systems, EHCI-DSVIS'2004, Kluwer Academic Press, pp 200-220 
Paternò FM (2001) Task models in interactive software systems. Handbook of Software Engineering and Knowledge Engineering

Paternò FM, Sciacchitano MS, Lowgren J (1995) A user interface evaluation mapping physical user actions to task-driven formal specification. In: Design, Specification and Verification of Interactive Systems, Springer Verlag, pp 155-173

Philipps J, Scholz P (1998) Formal verification and hardware design with statecharts. In: Prospects for Hardware Foundations, ESPRIT Working Group 8533, NADA - New Hardware Design Methods, Survey Chapters, pp 356-389

Puerta A, Eisenstein J (2002) XIML: A Universal Language for User Interfaces. In: Intelligent User Interfaces (IUI), ACM Press, San Francisco

Reeve G (2005) A refinement theory for $\mu$ charts. PhD thesis, The University of Waikato

Reeve G, Reeves S (2000a) $\mu$-Charts and Z: Examples and extensions. In: Proceedings of APSEC 2000, IEEE Computer Society, pp 258-265

Reeve G, Reeves S (2000b) $\mu$-Charts and Z: Hows, whys and wherefores. In: Grieskamp W, Santen T, Stoddart B (eds) Integrated Formal Methods 2000: Proceedings of the 2nd. International Workshop on Integrated Formal Methods, Springer-Verlag, LNCS 1945, pp 255-276

Reichart D, Dittmar A, Forbrig P, Wurdel M (2008) Tool support for representing task models, dialog models and user-interface specifications. In: Interactive Systems. Design, Specification, and Verification: 15th International Workshop, DSV-IS'08, Springer-Verlag, Berlin, Heidelberg, pp 92-95

Scholz P (1996) An extended version of mini-statecharts. Tech. Rep. TUMI9628, Technische Univerität München, URL http://www4.informatik.tumuenchen.de/reports/TUM-I9628.html

Thimbleby H (1990) Design of interactive systems. In: McDermid JA (ed) The Software Engineer's Reference Book, Butterworth-Heineman, Oxford, chap 57

Thimbleby H (2004) User interface design with matrix algebra. ACM Trans Comput-Hum Interact 11(2):181-236

Woodcock J, Davies J (1996) Using Z: Specification, Refinement and Proof. Prentice Hall 